\title{
Hidden transient chaotic attractors of Rabinovich-Fabrikant system
}

\author{
Marius-F. Danca
}

Received: date / Accepted: date

\begin{abstract}
In [1, it is shown that the Rabinovich-Fabrikant (RF) system admits self-excited and hidden chaotic attractors. In this paper, we further show that the RF system also admits a pair of symmetric transient hidden chaotic attractors. We reveal more extremely rich dynamics of this system, such as a new kind of "virtual saddles".
\end{abstract}

Keywords Hidden transient chaotic attractor; Hidden attractor; Self-excited attractor; Rabinovich-Fabrikant system

\section{Introduction}

Nowadays, the notion of self-excited and hidden attractor introduced by Leonov and Kuznetsov [2, 3, 4, has become a common subject (see e.g. [5, 6, 7, 8, 9, 10, 11, 12, 13, 14, 15, 16 ). The main characteristic of hidden attractors is that their basins of attraction do not intersect with arbitrary small neighborhood of any equilibrium point, while a basin of attraction of a self-excited attractor is associated with some unstable equilibrium. In this context, stationary points are less important for finding hidden attractors than for finding self-excited attractors. Self-excited attractors can be localized (excited) by standard computational procedures, by starting from a point in some neighborhood of an unstable equilibrium, while for localization of hidden attractors it is necessary to develop special numerical procedures. Some well-known classical chaotic and regular attractors (such as Loren ${ }^{1}$. Chen, Rösler, van der Pol, some Sprott systems, etc.) are self-excited attractors,

Marius-F. Danca

Department of Mathematics and Computer Science

Avram Iancu University, 400380 Cluj-Napoca, Romania

and

Romanian Institute for Science and Technology

400487 Cluj-Napoca, Romania

E-mail: danca@rist.ro

1 The possible existence in the Lorenz of a hidden chaotic attractor in the Lorenz system is an open problem [4. 
which can be localized numerically with standard computational procedures. However, as for the example considered in this paper, the system has both self-excited and hidden attractors. Hidden attractors are important in engineering applications because they may allow unexpected and potentially disastrous responses to perturbations in a structure like a bridge or aircraft wing. Hidden attractors can be found in systems with no equilibria [17, 18, or with only one stable equilibrium [19], which is a special case of multistable systems or coexistence of attractors [20]. Uncovering all co-existing attractors and their underlying basins represents one of the major difficulties in locating hidden attractors.

On the other hand, transient chaos, ubiquitous in chaotic systems, is due to nonattracting chaotic saddles in phase space [21, 22, 23, 24, 25, 26, 27, 28, 29, 30, 31, 32, 33, 34, 35]. The transient chaos represents a phenomenon which appears when a nonlinear system behaves chaotically during some (short or long, but finite) transient time after which falls into a periodic or chaotic attractor. Initially, the behavior is aperiodic and the system is sensitive to initial conditions (i.e. "chaos"), after which it settles down on a periodic orbit (or fixed point), or some chaotic attractor. Transient chaos is a common phenomenon of many engineering, physical and biological systems. Dynamics on systems with chaotic transients can be unpredictable even finally the system falls into a very simple motion. Chaotic transients can be found in systems with multiple (competing) attractors (as in the Rabinovich-Fabrikant (RF) system considered in this paper) and the existence of chaotic transients may have important implications for experiments on chaotic systems. For example, such transient phenomena were observed in hydrodynamics 32, radio circuits 31, neural networks 33, Lorenz system 34, Rössler system [36], maps [35, experiments [31] and so on. Note that transient chaos can be quite disastrous and therefore unwanted, and it can be the cause of catastrophic developments in a dynamic system, for example in situations of voltage collapse or species extinction 30. Therefore, control or anticontrol (in the sense of maintaining) of transient chaos can be desirable in some cases.

To note that in a recent paper [37] it is shown that a new phenomenon of doubly transient chaos, which is fundamentally different from the hyperbolic and nonhyperbolic transient chaos reported in the literature, appears in many other systems (chemical reactions, binary star behavior, etc.), and is likely far less predictable than what has been previously thought. Therefore, finding hidden transient chaotic attractors represents a new and interesting challenge.

In this paper, we investigate numerically hidden transient chaotic attractors in the RF system.

The rest of the paper is organized as follows: Section 2 presents briefly the RF system and the stability of equilibria, and Section 3 deals with hidden transient chaotic attractors of the RF system. Conclusion is drawn in the last section.

\footnotetext{
2 Generally, the lifetime of the transient could be extremely long (superpersistent) (see 25] where it is shown that the average lifetime of chaotic transients for two-dimensional maps could last tens of thousands of iterations and also scales with the system parameter variation).
} 


\section{The RF system}

The RF system considered here is a chaotic system modeled by the following system of ODEs 1

$$
\begin{aligned}
& \dot{x}_{1}=x_{2}\left(x_{3}-1+x_{1}^{2}\right)+a x_{1}, \\
& \dot{x}_{2}=x_{1}\left(3 x_{3}+1-x_{1}^{2}\right)+a x_{2}, \\
& \dot{x}_{3}=-2 x_{3}\left(b+x_{1} x_{2}\right),
\end{aligned}
$$

where $a>0$ and $b$ is the bifurcation parameter. The system, revealed numerically in [1], presents unusual and extremely rich dynamics, including multistability (coexistence of multiple attractors for a given set of parameters), which represents an important ingredient for potential existence of hidden attractor.

Due to the complexity of the ODEs (third-order nonlinearities), a complete mathematical analysis such as stability of equilibria, existence of invariant sets, existence and convergence of heteroclinic or homoclinic orbits, has to be done numerically (mostly investigated in [1]).

The equilibria are $X_{0}^{*}(0,0,0)$ and

$$
X_{1,2}^{*}\left(\mp x_{1,2}^{*}, \pm y_{1,2}^{*}, z_{1,2}^{*}\right), X_{3,4}^{*}\left(\mp x_{3,4}^{*}, \pm y_{3,4}^{*}, z_{3,4}^{*}\right)
$$

where

$$
\begin{aligned}
& x_{1,2}^{*}= \pm \sqrt{\frac{b R_{1}+2 b}{4 b-3 a}}, \\
& y_{1,2}^{*}= \pm \sqrt{b \frac{4 b-3 a}{R_{1}+2}}, \\
& z_{1,2}^{*}=\frac{a R_{1}+R_{2}}{(4 b-3 a) R_{1}+8 b-6 a},
\end{aligned}
$$

and

$$
\begin{aligned}
& x_{3,4}^{*}= \pm \sqrt{\frac{b R_{1}-2 b}{3 a-4 b}}, \\
& y_{3,4}^{*}= \pm \sqrt{b \frac{4 b-3 a}{2-R_{1}}}, \\
& z_{3,4}^{*}=\frac{a R_{1}-R_{2}}{(4 b-3 a) R_{1}-8 b+6 a},
\end{aligned}
$$

with $R_{1}=\sqrt{3 a^{2}-4 a b+4}$ and $R_{2}=4 a b^{2}-7 a^{2} b+3 a^{3}+2 a$.

We consider in this paper the case with $a=0.1$ and $b=0.279$. Thus, the equilibria $X_{1,2,3,4}^{*}$ are

$$
\begin{aligned}
& X_{1,2}^{*}=(\mp 1.1600, \pm 0.2479,0.1223), \\
& X_{3,4}^{*}=(\mp 0.0850, \pm 3.3827,0.9953) .
\end{aligned}
$$

The system exhibits the symmetry $E$

$$
E\left(x_{1}, x_{2}, x_{3}\right) \rightarrow\left(-x_{1},-x_{2}, x_{3}\right) .
$$

Under the transformation $E$, each trajectory has its symmetrical (twin) trajectory with respect to the $x_{3}$-axis. Therefore, one can consider the stability of $X_{0}^{*}, X_{1}^{*}$ and $X_{3}^{*}$ only. 
In order to find the hidden transient attractors, we need to determine the stability of all equilibria. The Jacobian is

$$
J=\left[\begin{array}{ccc}
2 x_{1} x_{2}+a & x_{1}^{2}+x_{3}-1 & x_{2} \\
-3 x_{1}^{2}+3 x_{3}+1 & a & 3 x_{1} \\
-2 x_{2} x_{3} & -2 x_{1} x_{3} & -2\left(x_{1} x_{2}+b\right)
\end{array}\right] .
$$

All equilibria are hyperbolic.

The eigen-spectrum at the equilibrium $X_{0}^{*}$ is $\Lambda=\left\{\lambda_{1}, \lambda_{2}, \lambda_{3}\right\}=\{-0.5580,0.1000-$ $1 i, 0.1000+1 i\}$. Thus, $X_{0}^{*}$ is a repelling focus saddle. Its two-dimensional unstable manifold $W_{X_{0}^{*}}^{u}=\left\{x_{3}=0\right\}$ (i.e. plane $\left(x_{1}, x_{2}\right)$ ) and the one-dimensional stable manifold $W_{X_{0}^{*}}^{*}=\left\{x_{1}=x_{2}=0\right\}$ (i.e. axis $x_{3}$ ), meaning that the trajectories close to $X_{0}^{*}$ move to the saddle along the axis $x_{3}$, but are rejected via spiralling in the plane $\left(x_{1}, x_{2}\right)$.

The equilibrium $X_{1}^{*}$ has the eigen-spectrum $\Lambda=\{-0.2470,-0.0555-1.4722 i,-0.0555+$ $1.4722 i\}$ and, therefore, $X_{1}^{*}\left(X_{2}^{*}\right)$ is a stable focus node. Therefore, all trajectories approaching $X_{1,2}^{*}$ are attracted by these equilibria.

For the equilibrium $X_{3}^{*}, \Lambda=\{0.1981,-0.2780-4.7739 i,-0.2780+4.7739 i\}$ and, therefore, $X_{3}^{*}\left(X_{4}^{*}\right)$ is an attracting focus saddle. All trajectories, arriving in close vicinities of the two-dimensional stable manifold of $X_{3,4}^{*}$ spanned by the pair of eigenvectors corresponding to the complex conjugate eigenvalues $\lambda_{2,3}=$ $-0.2780 \pm 4.7739 i$, are attracted via spiralling toward $X_{3,4}^{*}$. After that, they are rejected on the unstable direction of the eigenvector corresponding to $\lambda_{1}=0.1981$.

Therefore, equilibria $X_{1,2}^{*}$ are stable and equilibria $X_{0}^{*}, X_{3,4}^{*}$ are unstable.

\section{Hidden transient chaotic attractors}

The numerical integration of the RF system represents a real challenge to ODEs solvers. The numerical results depend drastically on the initial conditions, integration step-size, and even the numerical method. Therefore, for some values of the parameters, some available fixed-step numerical methods for ODEs, implemented in software packages, might give unexpectedly different results for the same parameter values and initial conditions. Contrarily, fixed-step-size schemes, such as the standard RungeKutta method (RK4), or the multi-step predictorcorrector LIL method [38, generally give more accurate results (see details in [1]).

As revealed in [1], the RF system presents extremely rich dynamics. In this paper, we are concerned with chaotic attractors only. As can be seen from Fig. 1. there exist different-shaped chaotic attractors and also coexisting chaotic attractors (Figs. 1 (d),(f)). In 39, it is shown that, the system presents two hidden chaotic attractors corresponding to $b=0.2715$ (Fig. 1 (a)) and $b=0.2876$ (Fig. 1) (b)). The other chaotic attractors (Figs. 1 (c),(d) and (f)) are self-excited. By physical reasons, the parameters $a$ and $b$ should be positive. However, also, negative values revealed interesting dynamics (see Fig. 1 (e), where $a=-1$ and $b=-0.1$ ).

We next show that the coexisting chaotic attractors $\left(H_{1}\right.$ and $H_{2}$ in Fig. 1 (f)), for $b=0.279$ and initial conditions $x_{0}= \pm(-0.1,0.1,0.3)$ (points $\left.S_{1,2}\right)$ are transient hidden chaotic attractors.

From a computational perspective and based on the connection of basins of attraction of attractors with equilibria in the phase space, the following classification of attractors is utilized in this work. 
Definition 1 [2, 3, 4] An attractor is called a self-excited attractor if its basin of attraction intersects with any open neighborhood of a stationary equilibrium; otherwise, it is called hidden attractor.

Due to the symmetry $E$, we only consider the attractor $H_{1}$.

The transient character of this attractor is revealed by the time series of the first component $X_{1}$, starting from the attraction basin of $H_{1}$ (Fig. 2 (a) green plot), compared to the time series of the stable component $x_{1}^{*}$, starting from a different initial condition (red plot).

As can be seen, the lifetime of the transient is about $T^{*} \approx 160 s^{3}$, after which the trajectory is attracted by the stable equilibrium $X_{1}^{*}$ (component $x_{1}^{*}$ in Fig. 2 (a)).

The chaotic behavior is underlined by the positiveness of the largest Lyapunov exponent (Fig. 2 (b)).

In order to show that $H_{1}$ is a hidden transient chaotic attractor, first we have to show that all trajectories starting from vicinities of unstable equilibria $X_{3,4}^{*}$ tend either to stable equilibria $X_{1,2}^{*}$, or to infinity. In Fig. 3 (a), the related dynamics are presented, including $H_{1}$ and the trajectories starting from neighborhoods of unstable equilibria.

As can be seen, unstable equilibria $X_{3,4}^{*}$ reject a part of the trajectories to a new kind of set, $Y_{1,2}^{*}$, called "virtual saddles" in [1. Only few numerical methods can reveal these "virtual saddles". We believe that these sets are not only "virtual", but they really exist, and represent a characteristic of this system.

Note that the equilibria $X_{3,4}^{*}$, which seem to generate these "attractors", have their own saddles sets. Unstable directions of $X_{3,4}^{*}$ become stable directions in $Y_{1,2}^{*}$. Also, note that the distance between $Y_{1,2}^{*}$ and $X_{3,4}^{*}$ along the $x_{2}$ axis is quite large, in the order of $10^{3}$ depending on the integration step size [1,39.

In order to observe more details of the shape of the transient hidden chaotic attractor $H_{1}$, consider the enlarge view in Fig. 3 (b). Here, beside $H_{1}$ (green plot), one can see trajectories starting from unstable equilibria (for clarity only 50 trajectories are plotted).

Trajectories starting from a $\delta$-neighborhood $V_{X_{0}^{*}}$ of unstable equilibrium $X_{0}^{*}$ (with $\delta$ in the order of $10^{-4}$ ) tend to infinity via $Y_{1}^{*}$ or $Y_{2}^{*}$ (see grey plots in the zoomed image of $V_{X_{0}^{*}}$ in Fig. 3 (c)). Because the plane $\left(x_{1}, x_{2}\right)$ is the unstable manifold of $X_{0}^{*}$, trajectories starting from $V_{X_{0}^{*}}$, are first attracted to this plane following the stable direction $x_{3}$, after which they are rejected via spiralling due the positive components of the real parts of the eigenvalues.

All trajectories starting from $\delta$-neighborhoods $V_{X_{3,4}^{*}}$ of $X_{3,4}^{*}$ (with $\delta$ in the order of $10^{-4}$ ) tend either to stable equilibria $X_{1,2}^{*}$ (red and blue plots respectively), or to infinity (black plot), via virtual saddles $Y_{1,2}^{*}$ (see also the zoomed vicinity $V_{X_{3}^{*}}$ in Fig. $3(\mathrm{~d})$ ). One can see that all trajectories exit $V_{X_{3}^{*}}$ via spiralling, due to the positive real parts of the complex eigenvalues $\lambda_{2,3}$ of $X_{3}^{*}$.

Summarizing, Definition 1 applies and one can conclude that $H_{1}\left(H_{2}\right)$ is a hidden transient chaotic attractor.

The second task is the localization of the hidden attractor. In 2, 40, an analyticalnumerical localization algorithm is presented. However, trial-and-error methods

\footnotetext{
3 To obtain $T^{*}$ in seconds, we can use a uniform partition of the integration time with step length corresponding to the integration step size $h$ supposed to be measured in milliseconds.
} 
could be used too. Thus, for finding $H_{1,2}$, one can utilize RK4 (or LIL algorithm) with step size 0.0001 and initial conditions $S_{1,2} x_{0}= \pm(-0.1,0.1,0.3)$ (Fig. 4 reveals the position of the initial point $S_{1}$ situated outside the unstable neighborhoods of equilibria $X_{0}^{*}$ and $\left.X_{3,4}^{*}\right)$. Moreover, the dimension of the attraction basin of $H_{1}\left(H_{2}\right)$ seems to be significantly smaller than the basins of the self-excited attractors. Another modality to uncover hidden attractors is presented in [5, where the localization is realized via perpetual points introduced in [4].

\section{Conclusion}

In this paper, we have shown numerically that the RF system presents a pair of coexisting chaotic attractors, which are transient hidden chaotic attractors, with the lifetime of the chaotic transient $T^{*} \approx 160 \mathrm{sec}$. The hidden character has been verified by mathematical definition. All trajectories starting from small neighborhoods of unstable equilibria $X_{0}$ and $X_{3,4}^{*}$ do not tend to $H_{1,2}$, but either are attracted by the stable equilibria $X_{1,2}^{*}$ or tend to infinity via a new kind of sets, the "virtual saddles" $Y_{1,2}^{*}$. Further studies on $Y_{1,2}^{*}$ will be the subject of future works. Also, studying the existence of chaotic saddles and boundary crisis in systems with transient hidden chaotic attractors, could be a good start to find if there are differences (e.g. the transient lifetime) between this kind of transient chaos and the transient chaos in the periodic windows in almost all of the chaotic systems.

Acknowledgements We thank the reviewers for their insightful comments and suggestions.

\section{References}

1. Danca, M.-F., Fečkan, M., Kuznetsov, N., Chen, G.: Looking More Closely at the Rabinovich-Fabrikant System. Int. J. Bifurc. Chaos 26(02), 1650038 (2016)

2. Leonov, G.A., Kuznetsov, N.V., Vagaitsev, V.I.: Hidden attractor in smooth Chua systems. Physica D 241(18), 1482-1486 (2012)

3. Leonov, G., Kuznetsov, N., Mokaev, T.: Homoclinic orbits, and hidden attractors. Eur. Phys. J.-Spec. Top. 224(8), 1485-1491 (2015)

4. Leonov, G.A., Kuznetsov, N.V.: Hidden attractors in dynamical systems. From hidden oscillations in Hilbert-Kolmogorov, Aizerman, and Kalman problems to hidden chaotic attractors in Chua circuits. Int. J. Bifurc. Chaos 23(1), 1330002 (2013)

5. Dudkowski, D., Prasad, A., Kapitaniak, T.: Perpetual points and hidden attractors in dynamical systems, Phys. Lett. A 379(40?41), 2591-2596 (2015)

6. Li, C., Sprott, J.C.: Coexisting Hidden Attractors in a 4-D Simplified Lorenz System. Int. J. Bifurc. Chaos 24(3), 1450034 (2014)

7. Shahzad, M., Pham, V.-T., Ahmad, M.A., Jafari, S., Hadaeghi, F.: Synchronization and circuit design of a chaotic system with coexisting hidden attractors. Eur. Phys. J.-Spec. Top. 224(8), 1637-1652 (2015)

8. Brezetskyi, S., Dudkowski, D., Kapitaniak, T.: Rare and hidden attractors in Van der Pol-Duffing oscillators. Eur. Phys. J.-Spec. Top. 224(8), 1459-1467 (2015)

9. Cafagna, D., Grassi, G.: Fractional-order systems without equilibria: The first example of hyperchaos and its application to synchronization. Chinese Phys. B 24(8), 080502 (2015)

10. Zhusubaliyev, Z.T., Mosekilde, E.: Multistability and hidden attractors in a multilevel DC/DC converter. Math. Comput. Simulat. 109, 32-45 (2015)

11. Li, C., Sprott, J.C.: Chaotic flows with a single nonquadratic term. Phys. Lett. A 378(3), 178-183 (2014) 
12. Wang, Z., Sun, W., Wei, Z., Zhang, S.: Dynamics and delayed feedback control for a 3D jerk system with hidden attractor. Nonlinear Dyn. 82(1), 577-588 (2015)

13. Chen, M., Li, M., Yu, Q., Bao, B., Xu, Q., Wang, J.: Dynamics of self-excited attractors and hidden attractors in generalized memristor-based Chua's circuit. Nonlinear Dyn. 81(1), 215-226 (2015)

14. Jafari, S., Sprott, J.C., Hashemi Golpayegani S.M.R.: Elementary quadratic chaotic flows with no equilibria. Phys. Lett. A 377(9), 699-702 (2013)

15. Molaie, M., Jafari, S., Sprott, J.C., Hashemi Golpayegani S.M.R.: Simple chaotic flows with one stable equilibrium. Int. J. Bifurc. Chaos 23(11), 1350188 (2013)

16. Jafari, S., Sprott, J.C.: Simple chaotic flows with a line equilibrium. Chaos, Solitons \& Fractals, 57, 79-84 (2013).

17. Wang, X., Chen, G.R.: Constructing a chaotic system with any number of equilibria. Nonlinear Dynamics, 71(3), 429-436, (2013).

18. Wei, Z., Wang, R., Liu, A.: A new finding of the existence of hidden hyperchaotic attractors with no equilibria. Math. Comput. Simulat. 100, 13-23 (2014)

19. Wang, X., Chen, G.R.: A chaotic system with only one stable equilibrium. Commun. Nonlinear Sci. 17(3), 1264-1272 (2012).

20. Sprott, J.C., Wang, X., Chen, G.R.: Coexistence of point, periodic and strange attractors. Int. J. Bifurc. Chaos 23, 1350093, (2013)

21. Kaplan, J.L., Yorke, J.A.: Pre-turbulence: a regime observed in a fluid flow model of Lorenz. Commun. Math. Phys. 67(2), 93-108 (1979)

22. Yorke, J.A., Yorke, E.D.: Metastable chaos: the transition to sustained chaotic oscillations in the Lorenz model. J. Stat. Phys. 21(3), 263-277 (1979)

23. Pianigiani, G., Yorke, J.A.: Expanding maps on sets which are almost invariant. Decay and chaos, Trans. Am. Math. Soc. 252, 351-366 (1979)

24. Nusse, H.E., Yorke, J.A.: A procedure for finding numerical trajectories on chaotic saddles. Physica D 36(1-2), 137-156 (1989)

25. Grebogi, C., Ott, E., Yorke, J.A.: Fractal basin boundaries, long-lived chaotic transients, and unstable-unstable pair bifurcation. Phys. Rev. Lett. 50, 935-938 (1983)

26. Grebogi, C., Ott, E., Yorke, J.A.: Crises, sudden changes in chaotic attractors, and transient chaos. Physica D 7, 181-200 (1983)

27. Tél, T.: Transient chaos, Directions in Chaos, Vol.3, ed. B.-L. Hao, D.H. Feng, J.M. Yuan, World Scientific, Singapore (1991)

28. Grebogi, C., Ott, E., Yorke, J.A.: Chaotic attractors in crisis, Phys. Rev. Lett. 48, 1507 (1983)

29. Kantz, H., Grassberger, P.: Repellers, semi-attractors, and long-lived chaotic transients. Physica D 17(1), 75-86 (1985)

30. Dhamala, M., Lai, Y.-C.: Controlling transient chaos in deterministic flows with applications to electrical power systems and ecology. Phys. Rev. E 59, 1646-1655 (1999)

31. Zhu, L., Raghu, A., Lai, Y.-C.: Experimental observation of superpersistent chaotic transients. Phys. Rev. Lett. 86(18), 4017-4020 (2001)

32. Ahlers, G., Walden, R.W.: Turbulence near Onset of Convection, Phys. Rev. Lett. 44, 445-448 (1980)

33. Chen L., Aihara, K.: Chaotic simulated annealing by a neural network model with transient chaos. Neural Networks 8(6), 915 ?930 (1995)

34. Vadasz, P.: Analytical prediction of the transition to chaos in Lorenz equations. Appl. Math. Lett. 23(5), 503-507 (2010)

35. Astaf'ev, G.B., Koronovskil, A.A., Hramov, A.E.: Behavior of Dynamical Systems in the Regime of Transient Chaos. Tech. Phys. Lett.+ 29(11), 923-926, (2003)

36. Dhamala, M. , Lai, Y.-C., Kostelich, E.J.: Analysis of transient chaotic time series. Phys. Rev. E 64, 056207 (2001)

37. Motter, A.E., Gruiz, M., Károlyi, G., Tél, T.: Doubly transient chaos: Generic form of chaos in autonomous dissipative systems. Phys. Rev. Lett. 111, 194101 (2013)

38. Danca, M.-F.: A multistep algorithm for ODEs. Dyn. Contin. Discr. Impul. Syst. B 13(6), 803-821 (2006)

39. Danca, M.-F., Kuznetsov, N., Chen, G.: Unusual dynamics and hidden attractors of the Rabinovich-Fabrikant system. arXiv:1511.07765 2 http://arxiv.org/pdf/1511.07765v2.pdf

40. Leonov, G.A., Kuznetsov, N.V., Vagaitsev, V.I.: Localization of hidden Chua's attractors. Phys. Lett. A 375(23) 2230-2233 (2011)

41. Prasad, A., Existence of Perpetual Points in Nonlinear Dynamical Systems and Its Applications. Int. J. Bifurc. Chaos 25, 1530005 (2015) 
8

Marius-F. Dance

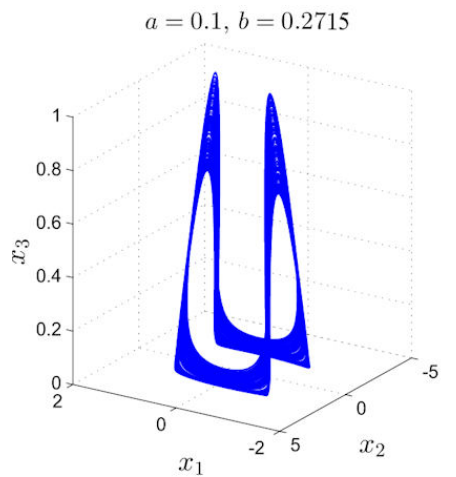

(a)

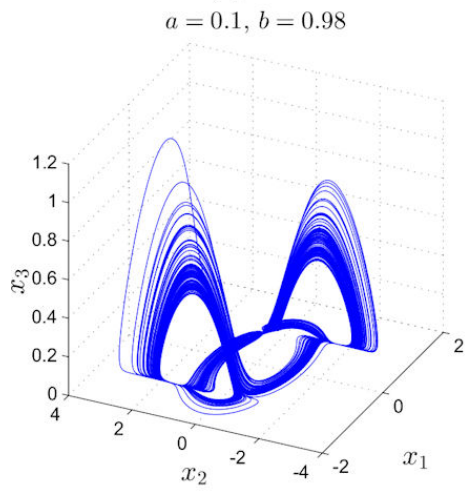

(c)

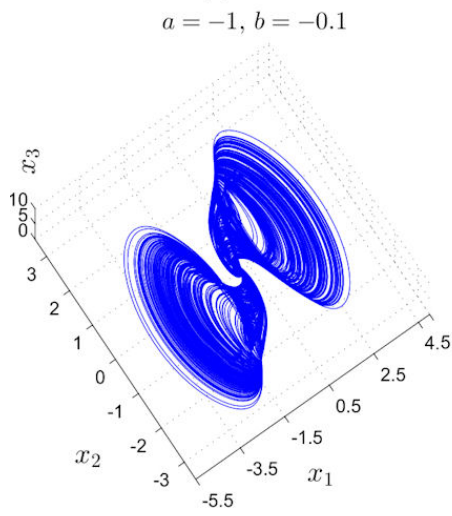

(e)

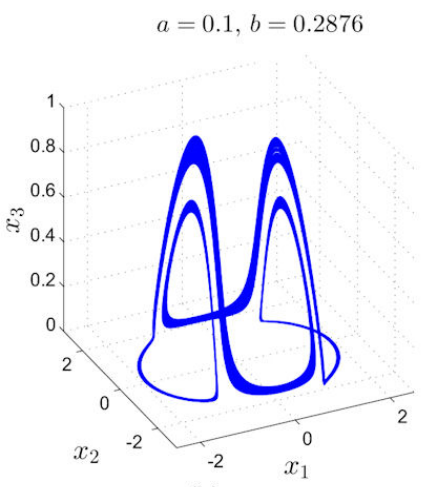

(b)

$a=0.1, b=1.22$

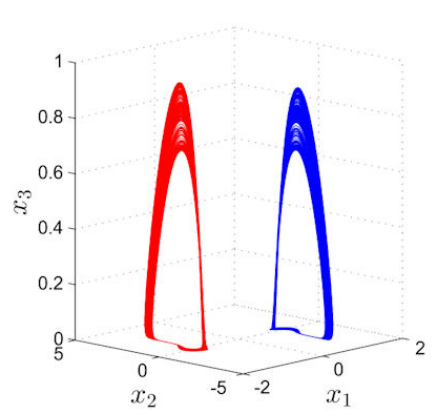

(d)

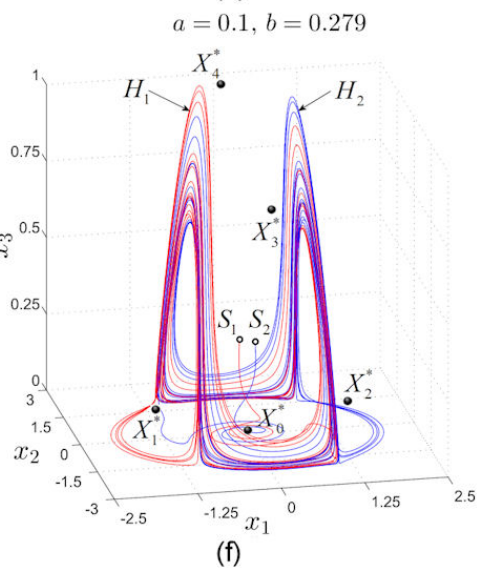

(f)

Fig. 1 Chaotic attractors of the RF system 11. (a), (b) Hidden chaotic attractors. (c) Selfexcited chaotic attractor. (d) Coexisting symmetric self-excited chaotic attractors. (e) Selfexciting chaotic attractor. (f) Coexisting transient hidden chaotic attractors $H_{1}$ and $H_{2}$. 


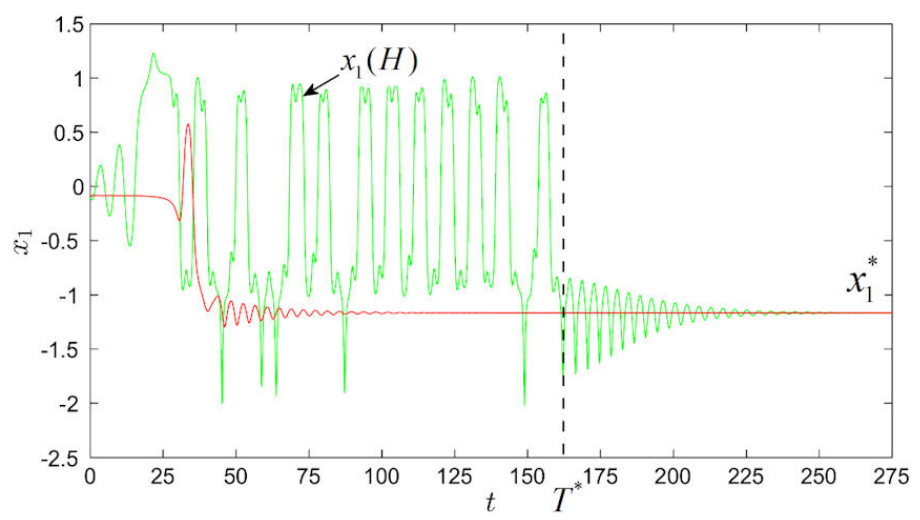

(a)

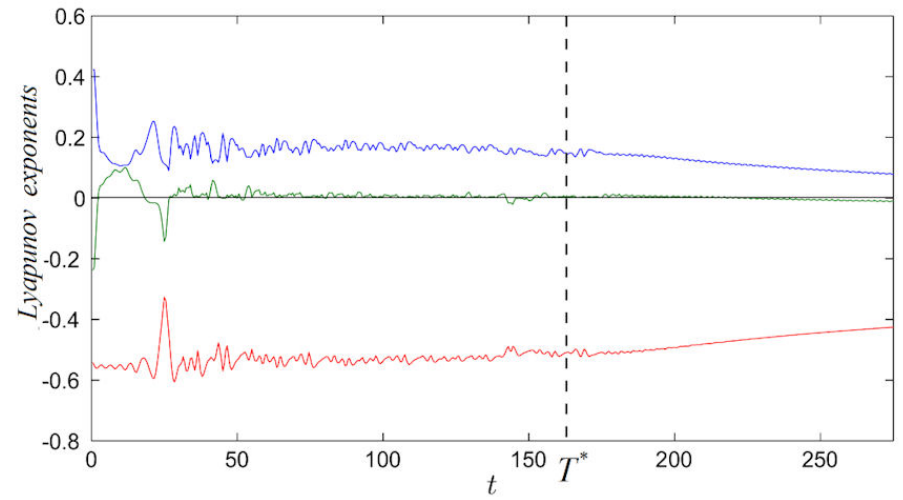

(b)

Fig. 2 (a) Time series of $x_{1}$ component of the transient hidden chaotic attractor $H_{1}$. (b) Lyapunov exponents of $H_{1}$. 


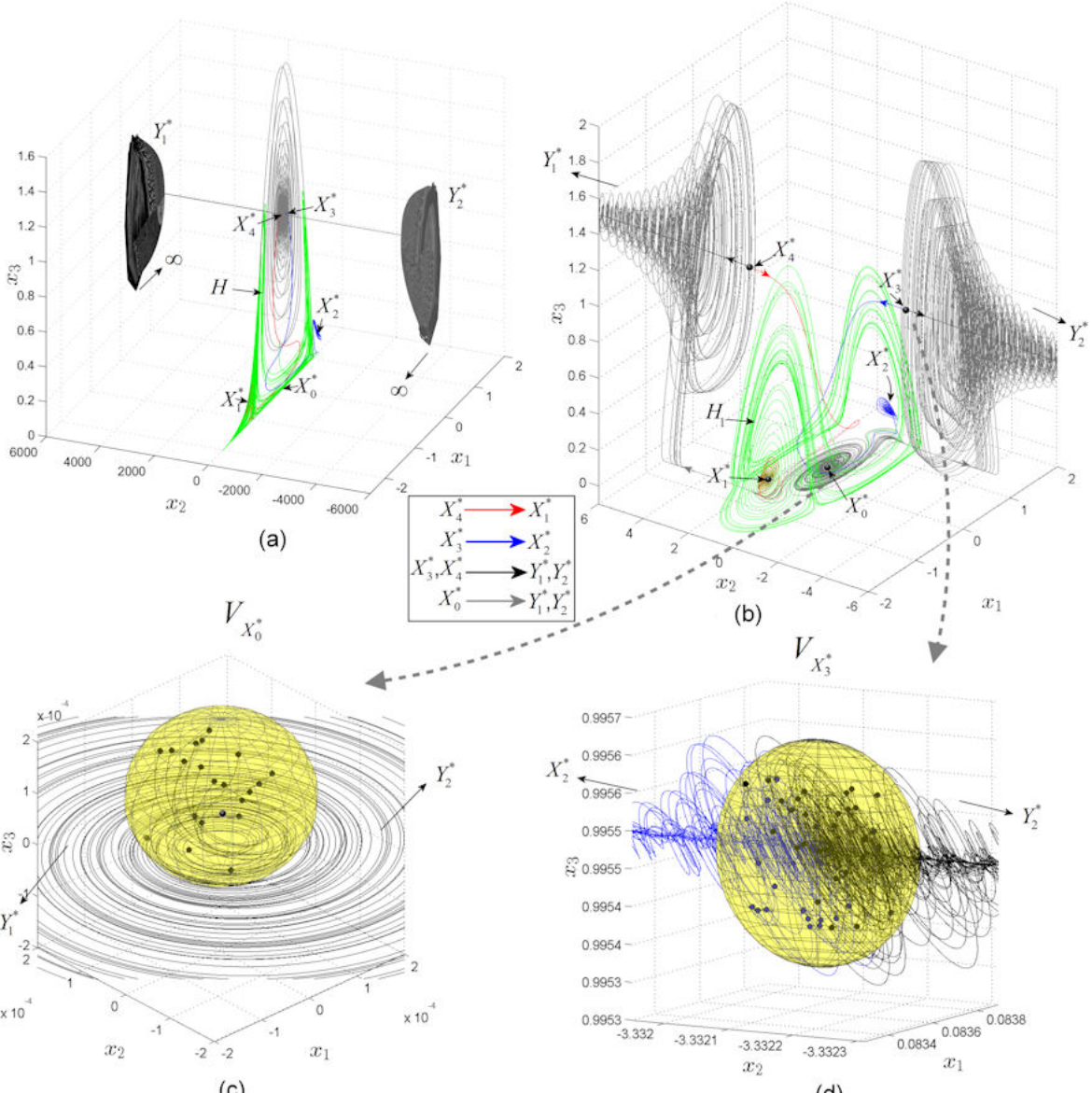

(c)

(d)

Fig. 3 Dynamics related to the transient hidden chaotic attractor $H_{1}$. (a) Hidden attractor $H_{1}$ and "virtual saddles" $Y_{1,2}^{*}$. (b) Zoomed image of the hidden attractor $H_{1}$. (c) Zoomed image of the neighborhood $V_{X_{0}^{*}}$ of the equilibrium $X_{0}^{*}$. (d) Zoomed image of the neighborhood $V_{X_{3}}$ of the equilibrium $X_{3}^{*}$. 


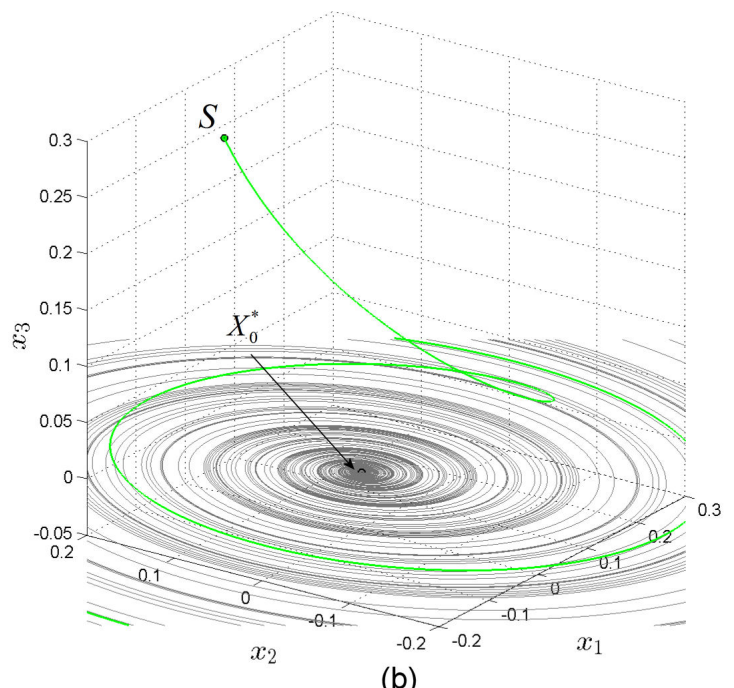

Fig. 4 Initial point $S$ of the transient hidden chaotic attractor. 